\title{
A coupled hydro - mechanical approach for modelling the volume change behaviour of compacted bentonite
}

\author{
Jose A. Bosch ${ }^{1, *}$, Alessio Ferrari ${ }^{1}$, and Lyesse Laloui ${ }^{1}$ \\ ${ }^{I}$ Swiss Federal Institute of Technology, EPFL, Station 18, Lausanne, Switzerland
}

\begin{abstract}
The volumetric response of compacted bentonites against environmental actions is a key aspect in most designs of nuclear waste repositories. The safety assessment of such repositories must account for robust and reliable models of stress-strain for bentonites. While many models for unsaturated low activity clays take advantage from the use of a generalized effective stress, its application to expansive soils has not found the same degree of success. One of the possible reasons is the complex water retention behaviour of these materials, which only recently has been successfully reproduced by numerical models. Here, by adopting an appropriate water retention model, a coupled hydro-mechanical approach to simulate the volume change behaviour of compacted bentonites is suggested. An explicit distinction between interlayer adsorbed water and capillary water is used to simulate the water retention behaviour. It is then shown that by using a precise water retention formulation, the volumetric behaviour can be interpreted within an effective stress-degree of saturation based framework. Some interesting results derived from the use of the effective stress include the shrinkage limit, the increase in stiffness of the elastic regime and the use of a single elastic coefficient for both wetting-swelling and reloading stress paths.
\end{abstract}

\section{Introduction}

It is widely accepted that two constitutive stress variables are needed to model the mechanical behaviour of unsaturated soils [1]. A comparison of different classes of stress frameworks for unsaturated soils was given in [2], concluding that generalisation of Bishop's definition of effective stress in a suitable elastoplastic framework, provides a number of conceptual and numerical advantages.

While for low activity soils the interest of an effective stress considering the hydraulic state has been well recognized [3, 4], most models intended for expansive clays do not consider the hydraulic state (e.g. [5]), generally given by the water retention curve, (WRC) on the mechanical response under unsaturated states, even though solid-water interactions are the salient feature of this class of material. Perhaps the main reason of this is that most effective stress expressions inevitably require a precise WRC model. In particular, bentonites can sustain high degrees of saturation at high suction values, hence a slight deviation of the simulated curve from reality can lead to a large deviation in the computed effective stress. Moreover, if the degree of saturation is adopted to model the increase of yield pressure in unsaturated states, the success of the mechanical model will directly depend on the WRC model predictions.

In this paper an accurate WRC formulation is used to study the suitability of using a single mechanical stress to be used in constitutive modelling of compacted expansive clays. The water retention of MX80 bentonite and the hydro-mechanical behaviour of Febex bentonite will be studied with the models presented.

\section{Modelling water retention behaviour}

In this section a similar model to that presented by Revil and $\mathrm{Lu}$ [6] is generalised to model the mechanisms of water retention of bentonite. For modelling purposes, water ratio $e_{w}$ (ratio of water volume with respect to volume of solids) is divided into capillary water ratio, $e_{w, \mathrm{c}}$ (volume of non-adsorbed water with respect to volume of solids) and adsorbed water, $e_{w, a}$ (volume of adsorbed water with respect to volume of solids):

$$
e_{w}=e_{w, c}+e_{w, a}
$$

And the degree of saturation is obtained as $S_{r}=e_{w} / e$. Although neglected in [6], the retention properties of capillary water are well known to be dependent on void ratio [7]. In the present work the evolution of capillary water ratio $e_{w, c}$ is modelled by the van Genuchten expression [8] modified by [9]:

$$
e_{w, c}=\left(e-e_{w, a}\right)\left[1+\left(a\left(e-e_{w, a}\right) b s\right)^{n}\right]^{1 / n-1}
$$

where $s$ stands for matric suction and $n ; a$ and $b$ are material parameters.

$e_{w, a}$ is a function of relative humidity following a Freundlich isotherm [13]. Expressed in terms of total suction it reads:

$$
e_{w, a}=e_{w, a}^{C}\left[\exp \left(M_{w} \psi d R T \rho_{\mathrm{w}, \mathrm{a}}\right)\right]^{1 / m}
$$

\footnotetext{
Corresponding author: jose.boschllufriu@epfl.ch
} 


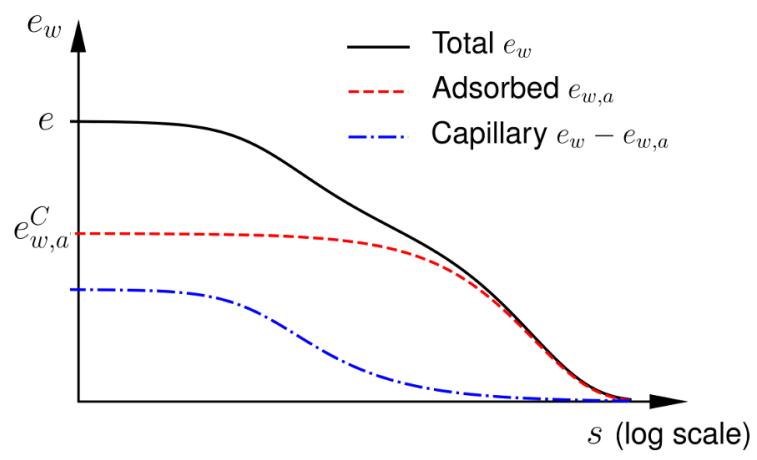

Fig. 1. Conceptual model of water retention including adsorption and capillary water.

Table 1. Calibrated values of water retention model parameters.

\begin{tabular}{|c|c|c|c|c|c|}
\hline Material & $n$ & $a\left(\mathrm{MPa}^{-1}\right)$ & $b$ & $e^{C}{ }_{w, a}$ & $m$ \\
\hline MX-80 & 1.8 & 1.5 & 1.5 & 0.55 & 1.2 \\
\hline Febex & 1.8 & 1.5 & 1.5 & 0.58 & 2.5 \\
\hline
\end{tabular}

where $e^{\mathrm{C}}{ }_{w, a}$ is a parameter that depends mainly on the type of exchangeable cations; $M_{w}$ is the molar volume of water; refers to total suction; $R$ is the universal gas constant; $T$ is the temperature; $\rho_{w, a}$ is the density of adsorbed water; and $m$ is a model parameter that depends mainly on the type of exchangeable cations. In cases in which osmotic suction can be neglected, Eq. (3) can be expressed as a function of matric suction, $s$ by replacing $\psi$ for $s$. This has been assumed in the model assessment cases presented in the following.

\subsection{Calibration of the water retention model}

The water retention model results are compared to experimental results from the literature. The comparison is made in terms of the measured values of water content $w=G_{s} e_{w}$; where $G_{s}$ stands for specific gravity (ratio between mass of solid particles to the mass of water).

The model has been first calibrated with MX-80 bentonite saturated under constant volume with experimental data reported by $[10,11]$ at different void ratios, as shown in Figure 2a. A good agreement with the three sets of data, can be obtained with values for the adsorption model that are in line with those reported in the literature.

In Figure $2 b$ the WRC obtained upon drying and wetting under free (unconstrained) volume conditions for FEBEX bentonite (experimental data from [12]) is shown. To calibrate the WRC parameters of the capillary model, the evolution of void ratio is needed for each measure, which were also reported in [12]. With respect to the WRC of the MX-80, only the adsorption parameters needed to be calibrated, which is reasonable given the different nature of the exchangeable cations.

A summary of the parameters determined by curve fitting is provided in Table 1.

\section{Modelling volume change of bentonite}

\subsection{Constitutive stress variables}

Only volumetric strains and mean stress states are considered in the following. Here we are interested in the definition of an effective stress in the sense that elastic strains are only due to changes in such effective stress. Many expressions for a single mechanical stress for unsaturated soils have been proposed, most of them being a particular form of the general expression proposed by Bishop [14]. Following the discussion by Nuth and Laloui [2] the suitability of.

$$
p^{\prime}=p^{\text {net }}+s S_{r}
$$

will be studied, where $p$ ' is the effective stress and $p^{\text {net }}$ is the net stress (excess of total stress over air pressure). This is a very practical form, since only the measurable water retention properties are needed for its determination.

The degree of saturation is used in addition to Eq. (7), in line with the approach proposed by Zhou et al. [15], to model the increase of compressibility under unsaturated conditions. The advantages of formulating constitutive equations in the $\left(p^{\prime}-S_{r}-e\right)$ space include a natural transition between unsaturated and saturated states upon compression. An additional advantage is that an air entry value parameter, needed to define the transition between saturated and unsaturated states when suction is used as a constitutive variable, is not needed, as saturated states are automatically defined when $S_{r}=1$. This is particularly advantageous for expansive soils because air entry value varies significantly due to large volume changes [16].

\subsection{Volume change model equations}

Constitutive equations are formulated according to the theory of elasto-plasticity whereby:

$$
\mathrm{d} \varepsilon_{v}=\mathrm{d} \varepsilon_{v}^{e}+\mathrm{d} \varepsilon_{v}^{p}
$$

where $\varepsilon_{v}$ is the volumetric strain and superscripts e and $\mathrm{p}$ stand for elastic and plastic respectively. The elastic part of deformation is given by:

$$
\mathrm{d} p^{\prime} / p^{\prime}=\mathrm{d} \varepsilon_{v}^{e} / \kappa
$$

where $\kappa$ is a material parameter defining the elastic compressibility.

Plastic strains develop while the isotropic yield condition $f=0$ is met:

$$
f=p^{\prime}-p^{\prime}{ }_{r}\left(p^{\prime}{ }_{Y S} / p^{\prime}{ }_{r}\right)^{\lambda_{s}-\kappa / \lambda(S r)-\kappa}
$$

where $p^{\prime}{ }_{r}$ is a reference stress at which yield pressure does not depend on $S_{r} ; s$ defines the elastoplastic compressibility during yielding for saturated states; $\left(S_{r}\right)$ is a function expressing the evolution of elastoplastic compressibility with the degree of saturation; and $p^{\prime}{ }_{Y S}$ is 

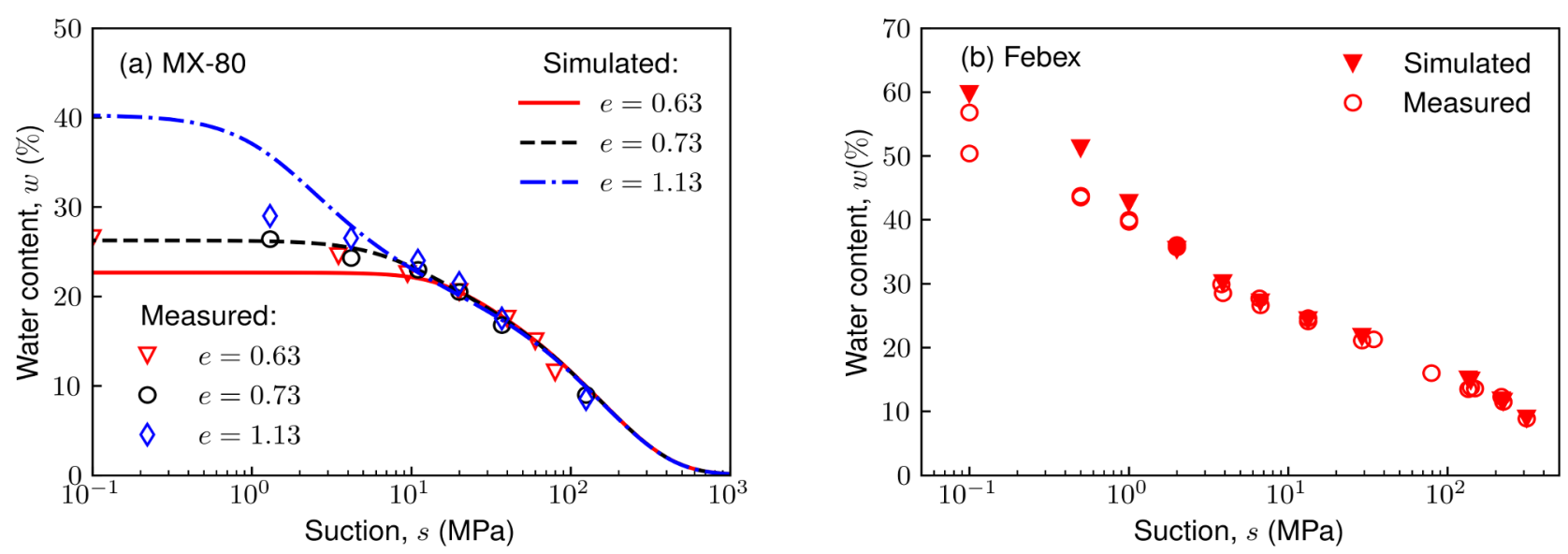

Fig. 2. a) Water retention calibration of MX80, (constant volume conditions) data from [10, 11]. b) Calibration of Febex bentonite water retention under free volume conditions with data from [12]; only discrete points can be computed as the evolution of void ratio is needed by the model.

the yield pressure at saturated state. $p^{\prime}{ }_{Y S}$ evolves according to the hardening law:

$$
\mathrm{d} p^{{ }^{\prime}{ }_{Y S}} / p^{\prime_{Y S}}=\mathrm{d} \varepsilon_{v}^{p} / \lambda_{s}-\kappa
$$

and the following expression is used for $\lambda_{s}(S r)$ :

$$
\lambda_{s}(S r)=\lambda_{s}-r\left(\lambda_{s}-\kappa\right)\left(1-S_{r}^{\zeta}\right)^{\xi}
$$

where the parameter $r$, bounded by $0<r<1$, expresses the decrease of plastic compressibility from saturated to dry state $\left(S_{r}=0\right)$; and $\zeta$ and $\xi$ are material parameters. An associated flow rule is used to compute volumetric plastic strains.

\subsection{Elastic response upon drying and wetting}

The volume changes of the FEBEX bentonite measured during the water retention test reported in [12] and reproduced in Figure $2 \mathrm{~b}$ is simulated. The test consisted in drying the material from an initial suction of $s=120$ MPa to $s=300 \mathrm{MPa}$, before proceeding to a wetting stage up to $s=0.1 \mathrm{MPa}$ by means of vapour control. A purely elastic behaviour is assumed for modelling this test, without external applied stress. Thus model equations (4-6) imply that:

$$
\mathrm{d} \varepsilon_{v}=\mathrm{d} e / 1+e=\kappa \mathrm{d}\left(s S_{r}\right) / s S_{r}
$$

hence volume changes are uniquely related to the water retention curve model and the parameter $\kappa$. In this case the water retention model has been calibrated in section 2. A sensitivity analysis of the influence of $\kappa$ on the resulting void ratio and water content is presented in Figure 3. Some interesting observations follow from this sensitivity study.

The shrinkage limit can be identified in the plane $(s-$ $e$ ). Upon drying, an asymptotic void ratio is approached which coincides with the experimental measurements and does not depend on the elastic modulus but on the water retention curve. This is due to the maximum effective stress, reached by the product $s S_{r}(s)$. In this regime, the value of $\kappa$ does not influence the results due to minor changes in effective stress.
Upon wetting below $s=100 \mathrm{MPa}$, volume changes depend on $\kappa$ and the measurements are satisfactorily matched by $\kappa=0.09$. Overall, the non-linear swelling response does not show a constant slope $\mathrm{d} e=\mathrm{d}(\ln s)$. Nevertheless, it is naturally reproduced by means of the effective stress with a constant $\kappa$. Unlike void ratio, water content only becomes dependent on $\kappa$ for relatively low values of suctions. Indeed, adsorption is the main mechanism by which water retention is modelled at high suction values and does not depend on void ratio.

\subsection{Suction controlled oedometric tests}

Lloret et al. [17] performed a series of suction controlled oedometric tests on Febex bentonite. Five tests were performed on samples that had the same initial compaction conditions with a void ratio of around 0.58 at a suction of around $127 \mathrm{MPa}$ (hygroscopic, ascompacted conditions). The samples were subjected to different stress paths including suction variations. The model presented will be used to simulate three of these tests (S3, S4 and S5 according to the notation in [17]), assuming isotropic stress conditions. the stress paths of the tests considered are reproduced in Figure 4. The objective is to verify that the single generalised effective stress can be used to interpret the responses under both suction and stress changes.

The parameter $\kappa$ was fitted to the deformation response upon wetting under constant stress of the test $\mathrm{S} 5$, while $s$ was found from the compression test under saturated states also from test S5. As already observed in the previous section the shrinkage limit upon drying at a suction of $500 \mathrm{MPa}$ (test S5) is well predicted by the effective stress formulation. Because oedometric conditions have been approximated to isotropic conditions for simulation purposes, the value of $\kappa$ cannot be expected to be the same as the one found in the sensitivity analysis shown in Figure 3, which reproduces a test under real isotropic conditions. Parameters $\zeta$, $\xi$ and $r$ were determined such that the wetting path of test S5 n 

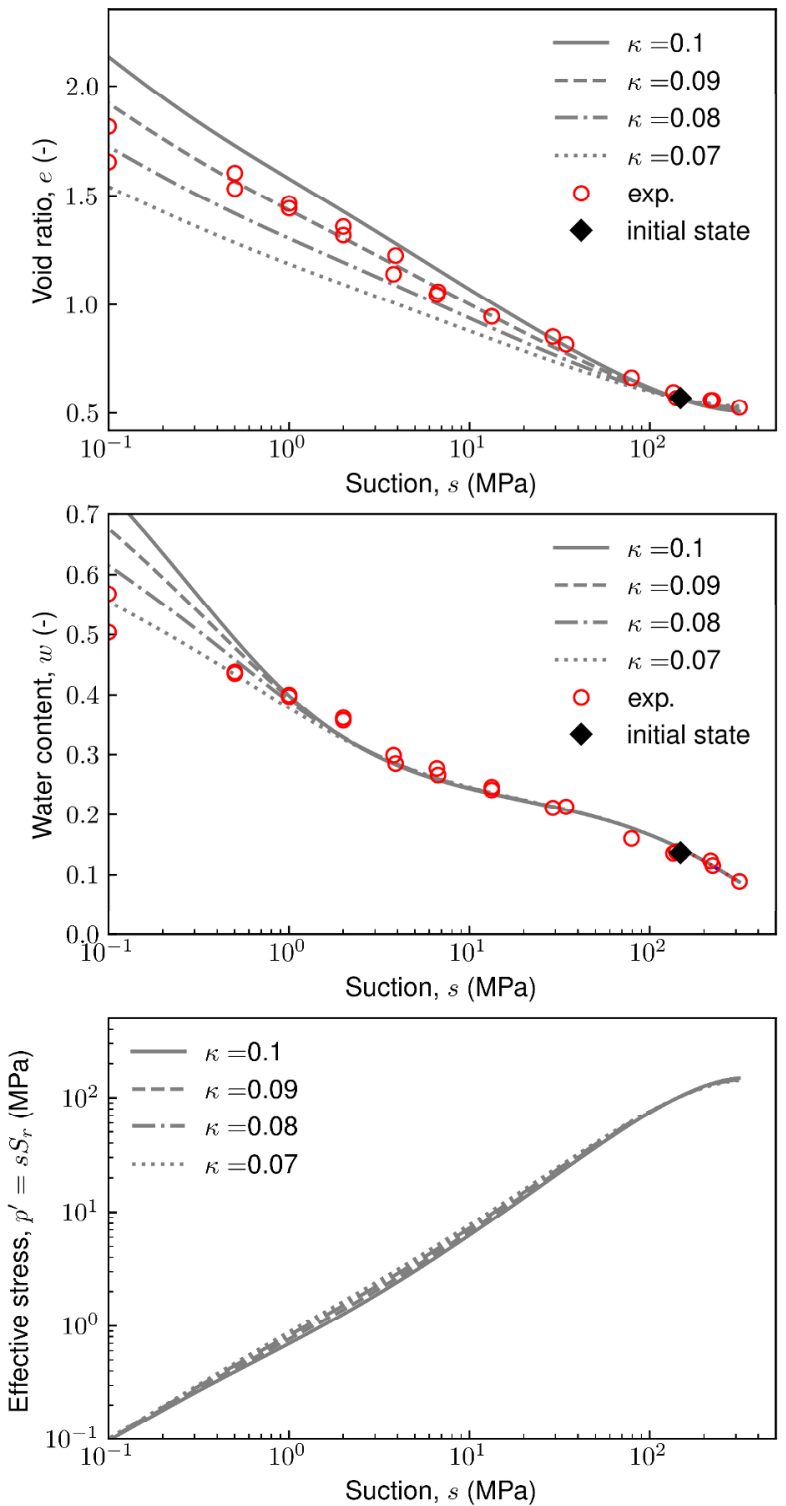

Fig. 3. Elastic model results for different values of for Febex bentonite dried and wetted under free volume conditions. Experimental data (denoted by exp.) from [12].

was fully elastic and that the yielding point of test S4 could approximate the experimental data. The complete set of model parameters calibrated is summarised in Table 2. As already mentioned, these parameters have been calibrated assuming isotropic stress-strain conditions. It is expected that if the real oedometric boundary conditions are established, different values will have to be used, particularly for $\kappa$ and $\lambda_{s}$.

Compression tests at a suction equilibrated of 14 and $4 \mathrm{MPa}$ (in tests S4 and S3) are therefore predictions. The responses upon compression of both $\mathrm{S} 4$ and $\mathrm{S} 3$ show a stiffness that is in accordance with the experimental results. Upon yielding, the non-linearity of the compression lines is interpreted by the model as a transition between different saturation states.

The suitability of the effective stress is also demonstrated when wetting of test $\mathrm{S} 3$ from a suction of 14 to $0.1 \mathrm{MPa}$ is simulated. In this case the swelling obtained is lower than that of test S5 for the same range of suctions due to the higher stress applied, in line with experimental results. It is worth noticing that while the elastic parameter, $\boldsymbol{\kappa}$ was determined from wetting (suction decrease path), the same value can match the loading-unloading elastic stages at different suction values. For Critical State models with pressure dependent bulk modulus, such as the present one, this variability in stiffness is an essential requirement for an effective stress [18].
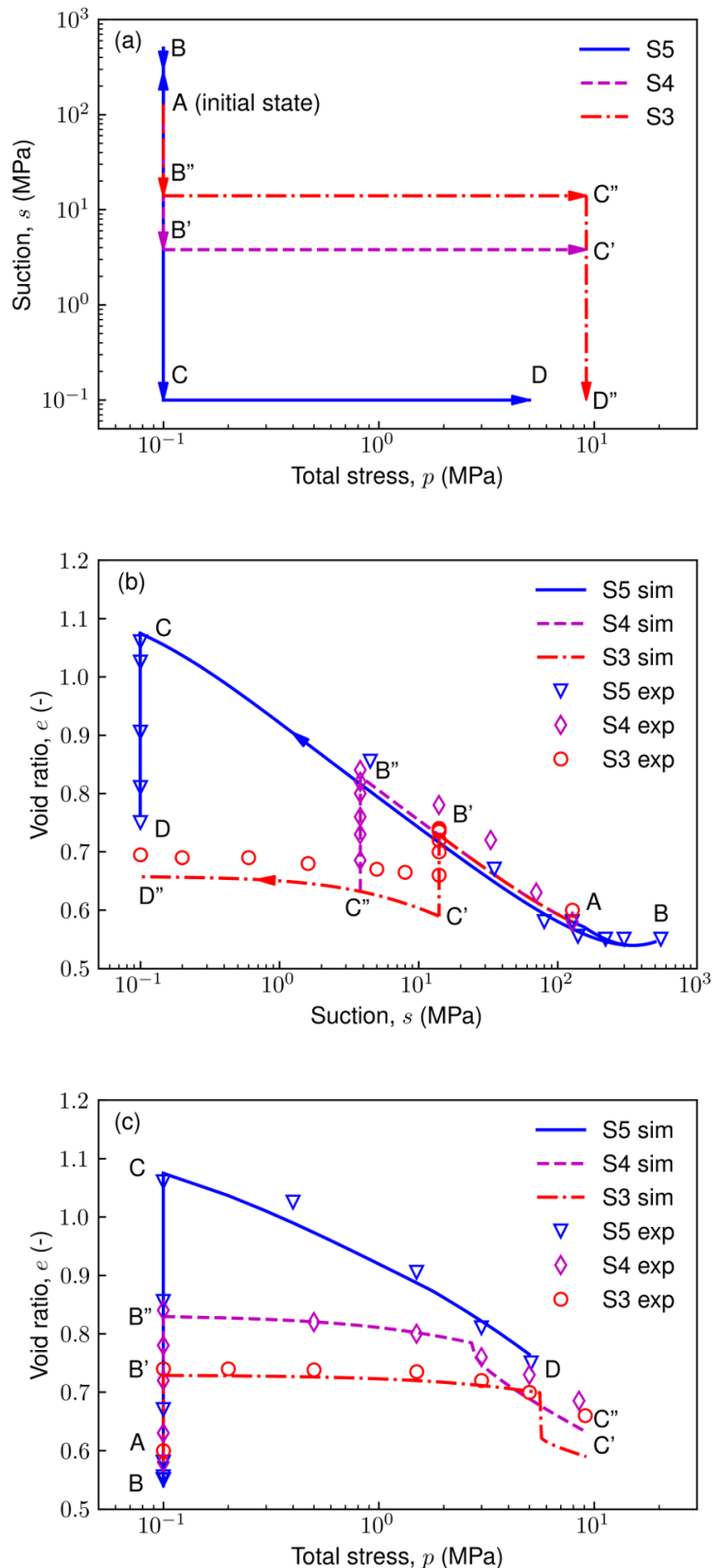

Fig. 4. Simulation of suction controlled oedometric tests on Febex bentonite. a) suction-stress paths of tests S3, S4 and S5 performed by [17]. B) Results in terms of suction-void ratio. c) Results in terms of total stress-void ratio. Model results are denoted by sim. While experimental data (from [17]) is denoted by exp. 
Table 2. Mechanical parameters calibrated for Febex bentonite.

\begin{tabular}{|c|c|}
\hline Parameter & Value \\
\hline$p^{\prime}{ }_{Y S}$ (initial) & $1.5 \mathrm{MPa}$ \\
\hline$\kappa$ & 0.045 \\
\hline$\lambda_{s}$ & 0.075 \\
\hline$r$ & 0.23 \\
\hline$\zeta$ & 20 \\
\hline$\zeta$ & 1.25 \\
\hline$p_{r}^{\prime}$ & $1 \mathrm{~Pa}$ \\
\hline
\end{tabular}

\section{Conclusions}

In this paper the coupled hydro-mechanical modelling ofcompacted bentonite by means of an effective stress has been explored. The use of a precise water retention model is a key requirement to use a generalised effective stress and the degree of saturation as constitutive variables for unsaturated soils. An explicit distinction between adsorbed water and capillary water provides a convenient framework to model the water retention behaviour of bentonites, provided that a dependency on volume is established. Furthermore, an isotropic mechanical model has been adopted to study the suitability of the degree of saturation as Bishop parameter in the definition of an effective stress. Model performance has been tested and compared with typical tests regarding volume change behaviour of bentonites. The shrinkage limit and free swelling of Febex bentonite is well captured by means of such effective stress. As required from an effective stress, the variability in stiffness at different degrees of saturation and the prediction of swelling upon wetting under different stresses are well predicted.

\section{Acknowledgments}

This work has received funding from the Euratom research and training programme 2014-2018 under grant agreement No 745942 .

\section{References}

1. A. Gens, Géotechnique 60, 3 (2010)

2. M. Nuth, L. Laloui, International journal for numerical and analytical methods in Geomechanics 32, 771 (2008)

3. S.J. Wheeler, R.S. Sharma, M.S.R. Buisson, Géotechnique 53, 41 (2003)

4. D. Gallipoli, A. Gens, R. Sharma, J. Vaunat, Géotechnique 53, 123 (2003)

5. A. Gens, E.E. Alonso, Canadian Geotechnical Journal 29, 1013 (1992)

6. A. Revil, N. Lu, Water Resources Research 49, 5685 (2013)

7. S. Salager, M. Nuth, A. Ferrari, L. Laloui, Canadian Geotechnical Journal 50, 200 (2013)
8. M. Van Genuchten, Soil science society of America journal 44, 892 (1980)

9. D. Gallipoli, S. Wheeler, M. Karstunen, Géotechnique 53, 105 (2003)

10. M.V. Villar, Informes Técnicos CIEMAT 1053, 39 (2005)

11. A.M. Tang, Y.J. Cui, Journal of Rock Mechanics and Geotechnical Engineering 2, 39 (2010)

12. M.V. Villar, Publicación técnica.(Empresa Nacional de Residuos Radiactivos) pp. 15-258 (2002)

13. H. Freundlich, akademische Verlagsgesellschaft (1909)

14. A.W. Bishop, Teknisk ukeblad 39, 859 (1959)

15. A.N. Zhou, D. Sheng, S.W. Sloan, A. Gens, Computers and Geotechnics 43, 178 (2012)

16. A. Seiphoori, A. Ferrari, L. Laloui, Géotechnique 64, 721 (2014)

17. A. Lloret, M. Villar, M. Sanchez, A. Gens, X. Pintado, E.E. Alonso (2003)

18. E.E. Alonso, J.M. Pereira, J. Vaunat, S. Olivella, Géotechnique 60, 913 (2010) 